\title{
Fazit: Klappe zu, Affe(kt) tot?
}

Zwei Jahre jeden Tag Affen; man wird schon selbst schimpansoid, und das wissenschaftlich Unangenehme: Es fällt einem nicht leicht mehr etwas an den Tieren auf. ${ }^{1}$

\subsection{Lektüren der Primatologie}

Die filmischen Bearbeitungen von Pierre Boulles La planète des Singes fallen durch ihre kreativen Eigenarbeiten und Ergänzungen auf. Häufig geht es dabei darum, die von Ulysse Mérou thematisierte Wissenslücke zu füllen: Wie kam es zum Niedergang der Menschheit und dem Aufstieg der Affenheit? Ob Atomkrieg, Sklaverei, genetische Manipulation und/oder Virus, die Bearbeitungen des von Boulle initiierten Stoffes bemühen sich redlich, diesen auszumalen - daher ist es umso auffälliger, welche Elemente des Urnarrativs sie aussparen: Hartnäckig haben die diversen Verfilmungen und Bearbeitungen ein wesentliches Konstruktionselement des Romans ausgeblendet: Ulysse Mérous Bericht von seiner Zeit auf dem Planeten der Affen erreicht uns nur, weil er im Roman bereits Leser/innen hat. Wie wir aus einer Rahmenerzählung erfahren, findet ein Paar auf Weltraumlustreise, „un couple de riches oisifs [...] parcouraient l'univers pour leur plaisir" (5), im All eine Flaschenpost und darin Ulysses Manuskript. Die Lektüre bewegt sie (,Certains passage de l'histoire l'avaient émue“, 191), ihr Inhalt erscheint ihnen aber unglaubwürdig. Denn dieses Paar ist ein schimpansisches, so stellt sich zum Schluss heraus:

Puis il commença de manœuvrer des leviers de commande, utilisant ses quatre mains agiles, tandis que Phyllis, ayant chassé un dernier doute en secouant énergiquement ses oreilles velues, sortait son poudrier et, en vue du retour au port, avivait d'un léger nuage rose son admirable mufle de chimpanzé femelle. (191)

\footnotetext{
${ }^{1}$ Wolfgang Köhler an Hans Geitel am 10.12.1915. In: Jaeger, Siegfried (Hg.): Briefe von Wolfgang Köhler an Hans Geitel 1907-1920. Passau 1988, S. 58.
} 
Menschliche Leser/innen lesen von Affen, die einen menschlichen Autor lesen, wie er Affen beobachtet, die wiederum Menschen beforschen, die Affen gleichen eine literarische Verkettung primatischer Lektüren.

Die vorliegende Studie gleicht dieser Verkettung. Sie las aus literaturwissenschaftlicher Perspektive die Texte von Forscher/innen, die Affen beforschen, und die nicht nur die Texte anderer Affenforscher/innen, sondern vielleicht auch Texte von Autor/innen gelesen haben, die die Texte von Autor/innen, die Affen beforschen, gelesen haben, und die manchmal von Affen handeln, die Menschen lesen können. Diese Verkettung kann verwirrend wirken, besonders hinsichtlich des epistemischen Gehalts der zugrunde liegenden Literatur. Anders als dies die Schimpans/innen Jinn und Phyllis für Ulysses Bericht annehmen (,Cela prouve qu'il y a des poètes partout, dans tous les coins du cosmos; et aussi des farceurs“, 191), stand bei allem Augenmerk für die Literarizität der primatologischen Forschungsmemoiren ihre Faktualität nicht infrage. Vor dem Hintergrund der Frage, welche Rolle die Affekte Forschender in der Feldforschung mit Affen spielen, wurde das Genre den Aussagen seiner Autor/innen und der Forschungsliteratur folgend als Medium betrachtet, in dem wahrheitsgetreu von realen Ereignissen und Erfahrungen berichtet wird:

Despite their value, scientific papers cannot express the fundamental charm, the fleeting social entanglement, the perishable moments of a baboon's life; they cannot deepen our love and understanding of another species, they cannot establish a heartfelt unity with creatures that were once part of our past. $^{2}$

Was ,scientific papers', wissenschaftliche Aufsätze, nicht können, vermögen die Forschungsmemoiren, wie George Schaller hier explizit macht: Sie eignen sich besonders für die Aufarbeitung emotionaler Erfahrungen im Feld und deswegen für eine umfassendere Abbildung der Forschungsarbeit. Oder, wie Shirley C. Strum es in ihrer „Author's Note“ zu Almost Human auf den Punkt bringt, „This book is fact, not fiction“" (xix).

Die größte Aufmerksamkeit im Feld der Primatologie-Rezeption lag bislang in der Forschungsliteratur auf den ,Trimates' als publikumswirksamen Gründerinnenfiguren der primatologischen Feldforschung. Auch in diesem Buch lag ebenfalls zunächst einen Schwerpunkt bei der Beschäftigung mit den Texten dieser Autorinnen. Dabei habe ich jedoch ein Verfahren zu vermeiden versucht, welches Torgovnick an der (auch wissenschaftlichen) Rezeption der ,Trimates“ Jane Goodall, Dian Fossey und Biruté Galdikas bemängelt hat: An drei weiblichen Primatologinnen, ihrer Forschungsarbeit und Emotionalität essentialistische Zuschreibungen zu den Affekten im Feld und gar den Emotionsprofilen von Geschlechtern finden zu wollen, eine Art Meta-Primatologie über

\footnotetext{
${ }^{2}$ Schaller, George: Foreword. In: Shirley C.: Strum: Almost Human. A Journey into the World of Baboons. New York/Toronto 1987, S. ix-xiii, hier S. xii.
} 
die Primatolog/innen und ihre Gefühle. ${ }^{3}$ Die vorliegende Untersuchung ging aus diesem Grund weit über die Episteme der ,Trimates' hinaus. Wie dabei gezeigt werden konnte, ist Emotionalität, sind ihre Ver- und Bearbeitung, ihre Bedingungen und Ausdrucksweisen durch sich historisch wandelnde äußere Umstände, kulturelle Bedingungen, wissenschaftliche Regularien aber auch die Gegenstände - unterschiedliche Affenarten, verschiedene Beobachtungsszenarien - zu variabel, als dass sich nach dem Muster „Wenn ein X-alter, kinderloser Mann ins Feld geht, um Affen zu untersuchen, dann fühlt er am Tag y Gefühl z. "4 Aussagen treffen ließen.

Das Anliegen der literatur- und kulturwissenschaftlichen Beschäftigung mit den Texten von Affenforscher/innen war also ein anderes als die Meta-Primatologie. Mittels der hier vollzogenen analytischen und kontextualisierenden Lektüren von primatologischen Forschungsmemoiren und Forschungsberichten aus einem fast ein Jahrhundert fassenden Zeitraum von 1917 bis 2012 ließen sich auf der Textbasis Aussagen treffen, die die eingangs aufgestellten Leitthesen belegen:

\section{Emotionalität spielt eine fundamentale epistemische Rolle in der Feldforschung} und bei der Generierung primatologischen Wissens. Frei lebende, ,wilde' Affen über längere Zeit in ihrem natürlichen Habitat zu studieren ist zunächst - bevor eine Infrastruktur des Forschens errichtet ist, deren sich heute Institute und Zentren für Affenforschung weltweit bedienen können - eine neue Aufgabe. Wie im ersten Teil der Studie, einer Untersuchung Affektiver Epistemologien des Feldes, diskutiert, knüpfen die Pionier/innen der Feld-Primatologie - die sogenannten ,Trimates“ Jane Goodall, Dian Fossey und Biruté Galdikas, aber auch der noch knapp vor Goodall im Feld arbeitende Zoologe George Schaller - in ihrer Feldforschung an ein bereits vorhandenes Wissen um die epistemischen Qualitäten von Emotionalität an, um diese Aufgabe zu bewältigen. Dieses Wissen scheint durch ein starkes wissenschaftliches Paradigma der Moderne, jenes der subjektbereinigten ,wissenschaftlichen Objektivität", wie es Lorraine Daston und Peter

\footnotetext{
${ }^{3}$ Torgovnick sieht die Meta-Primatologie vor allem im großen öffentlichen Interesse an diesen drei Forscherinnen formiert: Die ,Trimates“ „,have been ,gorilla-ized“. That is, they have been seen as offering clues to some essential truth about human, specifically female, sexuality. In the popular imagination, they were initially cast as lady scientists dutifully studying their subjects. But since her death, Fossey has been treated as though she herself were the subject of research whose habits would reveal the truth about female human nature. Female primatologists, as well as their apes, have been watched with prurient eyes". Torgovnick: Primitive Passions, S. 107.

${ }^{4}$ Noble zitiert einen auf diese Weise argumentierenden, anonym bleibenden kalifornischen Primatologen in Harold T.P. Hayes' Artikel für das Life Magazin (siehe Anm. 5 in Abschn. 2.5): „The very fact that the animal is so intelligent in human terms makes it difficult to withhold human response. This is particularly true with women researchers, and especially those who are childless. There comes an overwhelming need to protect. When this happens, look out, objectivity goes. The scientific credibility goes“. Noble: Politics, Gender, and Worldly Primatology, S. 545.
} 
Galison in ihrer Studie herausgeschält haben, zunächst verdeckt und verdrängt. ${ }^{5}$ Wie eine Untersuchung der Primärtexte dieser ersten Phase der Feld-Primatologie jedoch zeigt, wird der Zugang zu einem solchen Wissen durch die neue Forschungssituation provoziert und im Medium der Forschungsmemoiren auch theoretisierend explorierbar. Wie sich am ,Drama of Touch“, jener in Szene gesetzten Berührung von Mensch und Affe, die vor allem ein Strukturelement der frühen primatologischen Memoiren der ,Trimates' ist (aber vielleicht auch bei Schaller zu finden gewesen wäre, hätte er es hautnah an die Gorillas herangeschafft), zeigt, werden dabei ältere historische Gefühlskonzeptionen reaktiviert, etwa jene des Gefühls als haptisch gelenkter Erkenntnis, wie sie Bruno Gammerls lexika-

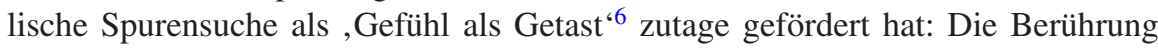
nicht etwas des Tieres, wie es das von Horkheimer und Adorno als Machtgeste interpretierte Streicheln eines (Haus-)Tieres wäre, ${ }^{7}$ sondern initiiert durch das Tier als Akteur/in in einer sozialen Beziehung, wird als affektives Indiz für eine gelingende Forschungssituation lesbar (Abschn. 2.2). Überraschenderweise lässt sich in den primatologischen Texten aber auch ein Gefühl finden, das am anderen Ende einer Skala von haptisch ,materieller" und distanzierter ,intellektueller" Affektivität steht: das Gefühl des Erhabenen als genuine Erkenntnisemotion. Es taucht in Texten auf, die von der Feldforschung mit Berggorillas in den Virungas berichten. Dieses Gefühl des Erhabenen in der Primatologie lässt sich erstens in der kantischen Tradition der überwältigenden Natur (hier: der Vulkanberge) als klassische ästhetische Emotion fassen. Zweitens wird in diesen Texten der Berggorilla selbst zum Tier des Erhabenen, an dessen Blickwechsel mit den Betrachter/ innen sich für diese Selbsterkenntnis erschließt, die drittens aber im Sinne der postmodernen Theorie des Erhabenen zu einer kritischen und sogar krisenhaften Reflexion führen kann. Viertens zeigt sich an der Gestaltung in der Literatur, wie an Lukas Bärfuss' Hundert Tage gezeigt, dass das Erhabene im Gorilla zum postmodernen Topos einer konsumierbaren egozentrischen Erfahrung geworden ist. Die Untersuchung des Erhabenen ,im Gorilla konnte damit auch illustrieren, wie sich das Gefühl des Erhabenen von Moderne zu Postmoderne wesentlich wandelt (Abschn. 2.3).

Der dem Gefühl des Erhabenen impliziten Ästhetisierung des Gorillas steht wiederum eine Vielfalt an affektiven Nahformen zur Seite, die die methodische Grundlage für die Feldforschung mit allen Affen bilden. Diese Nahformen der Anerkennung

\footnotetext{
${ }^{5}$ Vgl. Daston/Galison: Objectivity und die Einleitung dieser Studie, Abschn. 1.2.

${ }^{6}$ Gammerl: Gefühlte Entfernungen. Vgl. Abschn. 2.2.2.

${ }^{7}$, ,[D]as lässige Streicheln über Kinderhaar und Tierfell heißt: die Hand hier kann vernichten. Sie tätschelt zärtlich das eine Opfer, bevor sie das andere niederschlägt, und ihre Wahl hat mit der eigenen Schuld des Opfers nichts zu tun. Die Liebkosung illustriert, daß alle vor der Macht dasselbe sind, daß sie kein eigenes Wesen haben“. Horkheimer, Max/Adorno, Theodor W.: Dialektik der Aufklärung. In: Horkheimer, Max: „Dialektik der Aufklärung “ und Schriften 1940-1950. Hg. von Gunzelin Schmid Noerr. Frankfurt a. M. 1987 (Gesammelte Schriften, Bd. 5), S. 11-290, hier S. 285.
} 
provozieren eine Auseinandersetzung mit der anthropologischen Differenz, indem sie aus Objekten der Forschung Subjekte in einer Forschungsbeziehung zwischen Affe und Mensch zu den Bedingungen des Tieres werden lassen - im Gegensatz zur Laborforschung, die Tiere zu menschlichen Bedingungen untersucht (Abschn. 2.4). Die primatologische Feldforschung der ,ersten Stunde' nimmt damit eine Position zwischen zwei traditionellen affektiven Modellen der Affenbegegnung ein, wie sie im nüchternen Natur-Sammler Alfred Russel Wallace und dem sentimentalen Jäger Paul DuChaillu repräsentiert sind. Bezeichnenderweise waren bereits für diese traditionellen Affenbegegnungsmodelle spezifische affizierende Begegnungen - mit Tierjungen - Wendepunkte, weil sie die Affektmuster der Jagd und der Sammlung durch die längerfristige Habituation und Interaktion aufbrachen (Abschn. 2.1). Die Feldforschung verwirklicht das Affizierungspotenzial des Affen als Figur anthropologischer Selbsterkenntnis, geht dabei aber über die alleinige epistemische Nutzung des Tieres hinaus: Sie verleiht den Affen in den von ihnen handelnden Texten und mit einer Methodik, die auf Verfahren der Anerkennung beruht und die affektive Nähe zum Tier sucht, Subjektivität bzw. Agency. Affektive Beziehungen über die Tier-Mensch-Grenze hinweg provozieren jedoch gerade im Rahmen einer wissenschaftlichen Formation, die sich auch im 21. Jahrhundert noch aus der Erforschung der anthropologischen Differenz speist, Verdachtsmomente der Unwissenschaftlichkeit und - im fiktionalen Rahmen - der ,Interspecies Romance' mit ihrer doppelten Implikation der sentimentalen Romantisierung und der sexuellen Zoophilie, wie sie in Peter Goldsworthys Roman Wish und Michael Apteds Film Gorillas in the Mist exploriert wurden (Abschn. 2.5).

2. Die Wissensproduktion, wie sie dann in der wiedergebenden Darstellung der Feldforschung und der Lektüre von Affenarten und -gesellschaften erfolgt, korrespondiert mit den emotionalen Erfahrungen, wie sie sich in den Forschungsmemoiren qua Genre- bzw. Form-Auffälligkeiten bemerkbar machen. Der zweite Teil der Studie ging hinsichtlich der Affektpoetik der Forschungsmemoiren der Frage nach, wie die emotionalen Erfahrungen im Feld in die Gestaltung des Berichtes von diesen Felderfahrungen einfließen. Der Zusammenhang von Form und Inhalt, so zeigte sich, ist vielleicht nicht derart ,polymorph pervers “ wie der Überaffenmensch Tarzan in Edward Saids Einschätzung, ${ }^{8}$ dennoch aber komplex und vielfältig. Die Darstellung anhand von Genre als ,a tendential mode whereby signifying practices are organized" ${ }^{\text {"9 }}$ lässt sich grundsätzlich mit der Genre-Forschung als Verarbeitungs- und Ordnungsprozess irritierender Erfahrungen in der Überführung in Text verstehen (Abschn. 3.1). Verschiedene Genres, Formen oder Modi in Passagen der Forschungsmemoiren erfüllen dabei unterschiedliche Zwecke im Rahmen solcher Prozesse. So dient der melodramatische Modus, der sich in der schaulustigen Gestaltung individueller tierlicher Lebensgeschichten und Figuren und vor allem von Szenen des sexuellen Kontaktes bemerkbar macht,

\footnotetext{
${ }^{8}$ Siehe Said: Jungle Calling.

${ }^{9}$ Freedman: Critical Theory, S. 20.
} 
der normativierenden (Ein-)Ordnung der teilweise verstörenden Beobachtungen, der Generierung von Lesbarkeit der Affenfiguren und des primatischen Soziallebens für die Leser/innen sowie der Durchführbarkeit eigener hermeneutischer Verfahren. Er ermöglicht jedoch auch die aus der Doppelrolle als beobachtendes, kulturell ,programmiertes' Subjekt und professioneller, datensammelnder Wissenschaftler/in erwachsende Emotionsarbeit sowie eine subtile Bewertung des Dargestellten durch die melodramatische Darstellung selbst, bspw. indem auf das Repertoire an Plots und Figuren der ,female victimhood ' zugegriffen wird, die in Spannung mit den Normativierungsbemühungen stehen (Abschn. 3.2).

Besonders an eindeutig problematischem Affenverhalten in zeitlicher Koinzidenz mit negativen Erlebnissen in der Forschungssituation zeigt sich (am Beispiel von Jane Goodalls umfangreicher Publikation von Forschungsmemoiren), wie neues, bisherige theoretische Annahmen radikal in Frage stellendes, und eingeübte Affektlogiken (ver-)störendes Wissen - um das Vorhandensein von Kannibalismus und Auslöschungskrieg unter Schimpansen - dargestellt, normiert oder pathologisiert und dadurch letztlich klassifiziert wird. Dies geschieht durch den Einsatz rhetorischer Mittel (bspw. die Hypotypose der Zeugenschaft) und den Rückgriff auf literarische Formen (Kriegsbericht, Fallgeschichte, Trauma-Erzählung), die jeweils eigene Implikationen der Diskursivierung transportieren und Affenverhalten nach einer Ausrichtung auf die Pole ,normal ' und , abnorm ‘ hin modellieren (Abschn. 3.3).

Wieder andere Formen, die die primatologischen Forschungsmemoiren adaptieren, haben inhärent politisches und kritisches Potenzial, wie über mehrere Texte hinweg in Bezug auf den Nachruf, die Klage und Tropen der Trauer gezeigt werden konnte. Der Nachruf auf ein verstorbenes Forschungstier wird erst in dem Moment notwendig, in dem dieses Tier in einer sozialen Beziehung mit den Forschenden stand und/oder von existenzieller Bedeutung für das Forschungsprojekt war. Wirksam wird der Nachruf damit als Medium, das Tier zumindest im Tod als Subjekt in Erinnerung zu rufen, dessen Sterben einen persönlichen, aber auch wissenschaftlichen Verlust darstellt (Abschn. 3.4.2). Mit der weltweiten Gefährdung der Affenpopulationen durch Habitatdezimierung und Wilderei wird die melancholische Klage in der Primatologie aktiviert, die auf das Muster der Natur- und Artenelegie, wie sie Ursula Heise und Benjamin Bühler beschreiben, rekurriert und wie diese kritisches Potenzial in eine affektive politische Form gießt. ${ }^{10}$ Die Anwendung dieser Formen in den Forschungsmemoiren lässt auf die emotionale Verstrickung und das politische Engagement der Forschenden ebenso schließen wie auf den rhetorischen Gestus des Genres der Forschungsmemoiren selbst, das nicht nur von der Forschung berichten und unterhalten will, sondern auch für ein Anliegen argumentiert: Natur- und Artenschutz als Grundlage für weitere Forschung mit Affen - und für das ,Heil ' der postparadiesischen Menschheit, die sich in den immer weniger werdenden (Menschen-)Affen selbst betrachtet (Abschn. 3.4.3).

\footnotetext{
${ }^{10}$ Siehe Heise: Nach der Natur; Bühler: Ecocriticism.
} 
Die Formgebung emotionaler Erfahrungen in den Texten hat also, zugespitzt formuliert, drei Funktionen: Sie ist erstens Verarbeitungsprozess eigener, häufig irritierender neuer Erfahrungen auch hinsichtlich einer ,Lesbarkeit ${ }^{\text {" durch }}$ das Laienpublikum und Lokus von Emotionsarbeit; zweitens Klassifizierung von Affenverhalten hinsichtlich von epistemischem Verständnis und Vergleichbarkeit; drittens Raum der Auseinandersetzung, der Kritik und des Appells.

3. Die Darstellbarkeit, Thematisierbarkeit - und vielleicht auch Wahrnehmbarkeitvon Emotionen in der Forschung unterliegt den Schwankungen und der Rhetorik von wissenschaftlich, kulturell, gesellschaftlich und/oder politisch bedingten historischen Gefühlskonjunkturen. Die Thematisierung einer affektiven Epistemologie und die Genre-Darstellungsweisen emotionaler Erfahrungen und Beobachtungen im Text bewegen sich immer vor dem jeweiligen gesellschaftlichen, politischen, kulturellen und/oder wissenschaftlichen historischen Hintergrund für die einzelnen Forschungsmemoiren und ihre Autor/innen. Die Primatologie besteht zudem ihrerseits durch die Schulenbildung und interdisziplinäre Verfasstheit aus unterschiedlichen Denkkollektiven. Dieser im weitesten Sinn ,ideologische' Hintergrund (bzw. seine Teilbereiche), die Denkkollektive und die jeweiligen Gefühlskulturen prägen die den Text beherrschenden Affektregime der Primatologie. Die Bedingungen, unter denen primatologische (Feld-)For-schung und Theoriebildung stattfindet, bestimmen kurz gesagt die Äußerungsmöglichkeiten und Textstrategien. So zeigte sich, dass bspw. die Abwesenheit von Emotionalität auf semantischer Ebene des Vokabulars nicht für oder gegen eine subjektive Emotionalität der Autor/innen sprechen muss, sondern vor allem davon zeugt, in welchem wissenschaftstheoretischen und gesellschafts- bzw. wirtschaftspolitischen Paradigma ein Forschungsbericht entstanden ist - ob er etwa der Anthropomorphismus- und Anekdotenfeindlichkeit eines physiologisch fundierten Behaviorismus gerecht werden muss oder entemotionalisierte Theoriemodelle einer neoliberalisierten Soziobiologie nutzen kann (Abschn. 4.1 und 4.2). Auch die Möglichkeit, überhaupt eine den in den ersten beiden Teilen der Arbeit untersuchten emotionalen Erfahrungen vergleichbare Affektivität in der Feldforschung zu erleben, hängt von den Produktionsbedingungen primatologischen Wissens ab. Wie sich an den jüngsten Forschungsmemoiren der gegenwärtigen Primatologie zeigt, haben sich das ,Setting ‘ für die Forschung im Feld und die Methoden der primatologischen Wissensgenese derart verändert, dass die emotionale Erfahrung am Tier, wie sie Schaller, Goodall, Fossey, Strum, Galdikas und auch der nachfolgenden Generation noch offen stand, zumindest für die gegenwärtige Forschung mit Menschenaffen selten geworden ist. Das Genre der Forschungsmemoiren ist jedoch auch dadurch bestimmt, Medium für die Vermittlung dieser spezifischen Form der Emotionalität, der Forschungsemotionalität, zu sein. Daher stoßen Genre-Konventionen, die Affektivität und Affizierung implizieren, auf einen Mangel in der Praxis - und produzieren Strategien der Authentizitätskonstruktion im Text, die diesen von den Texten der Vorgänger/innen unterscheiden (Abschn. 4.4). Wie sich an Volker Sommers und Robert Sapolskys Texten zeigte, spielen aber auch literarische Prägungen eine Rolle für die Gestaltung und die Strategien im Text: Sommer bedient 
sich großzügig beim Vorbild des Afrikareiseberichts, um den Konventionen der Forschungsmemoiren entgegenzukommen (Abschn. 4.4.2). Sapolsky spielt mit den Charakteristika postmoderner Literatur, um durch Verfahren der Komik und der Ironisierung eine Distanz zwischen (auto-)biographischem und Gegenwarts-Ich, zwischen affektiver Rolle im Feld und kommentierender Rolle des Erzählers zu generieren, die ihm letztlich die politische Immunität verleiht, nach Belieben zu kritisieren, aber auch zu ,sentimentalisieren " - und seine Affen sogar stärker, als sich dies ältere (und neuere) Texte getraut haben, $\mathrm{zu}$ anthropomorphisieren (Abschn. 4.3).

Dieser letzte Punkt ist wichtig. Denn durch die Texte und ihre Unterschiede hindurch lässt sich als Gemeinsamkeit zugleich die Problematisierung von Anthropomorphismen und deren Anwendung finden (Abschn. 4.1). Letzteres ist der Verfasstheit menschlichen Denkens zuzurechnen, das per se epistemischen Anthropomorphismus praktiziert. Ersteres aber ist noch immer den strengen Normen eines positivistischen Ideals wissenschaftlicher Objektivität geschuldet, das von der Möglichkeit der Beseitigung aller Subjektivität und der damit einhergehenden Denkstrukturen ausgeht. Dabei stellt schon George Schaller im Vorwort zu Shirley C. Strums Forschungsmemoiren Almost Human fest:

If a scientist takes too much vocal pride in objectivity, beware. Observing is subjective: the animal described is only an illusion created out of a personal perspective, based on which questions are raised, which facts are written down, which information ignored. Another biologist asking different questions will create a different animal. The conspicuous, easily described behavior is turned into statistics; the difficult but no less real behavior tends to be ignored or considered irrelevant. To describe another being takes not merely reason and fact, but also empathy and intuition. ${ }^{11}$

Deutlicher ließe sich das Programm einer produktiven wissenschaftlichen Subjektivität in der Ethologie und die empirische Konstruktion des Forschungstieres kaum umreißen.

\section{Neben den Forschungsmemoiren liefert die Fiktion, sei dies Literatur oder Film,} einen eigenen Raum der Wissensproduktion und Reflexion für die Primatologie. Das obige Zitat George Schallers verweist in seiner Thematisierung der Absurdität der Objektivitäts-/Subjektivitäts-Dichotomie auf die Literarizität der Forschungsmemoiren und zugleich auf die Reflexionsmöglichkeiten, die den Autor/innen aus der schriftlichen Bearbeitung ihrer Erfahrungen und der Forschungsergebnisse in dieser Form erwachsen. Dieses, literarische' Programm setzt sich in der Bearbeitung der Primatologie durch die fiktionale Literatur selbst produktiv fort: Das kreative epistemische Potenzial der Literatur für die Primatologie liegt in ihrer Freiheit von wissenschaftlichen Normen, Objektivitätsgeboten oder auch nur Realitätsgebundenheit. Die primatologisch inspirierte literarische ,Science/ Fiction' operiert im Rahmen der Science-Fiction-Definition meist nur mit einem

\footnotetext{
${ }^{11}$ Schaller: Foreword, S. xi.
} 
veränderten Element: ${ }^{12}$ etwa mit der Möglichkeit genetischer Manipulation (in Peter Goldsworthys Wish) und rapider Evolution (in Benjamin Hales Bruno Littlemore und im Planet-der-Affen-Stoff), mit nicht-existenten Spezies (in Edgar Rice Burroughs' Tarzan oder Michael Crichtons Congo). Überwiegend aber zeigt sich die fiktionale Literatur der primatologischen Realität auffällig verbunden (so in William Boyds Brazzaville Beach, Ulrike Draesner Sieben Sprünge vom Rand der Welt und Bettina Suleimans Auswilderung). Vor dem Hintergrund dieser Realität bietet sich die Fiktion, wie sie in dieser Studie an zentralen Beispielen diskutiert wurde, der Primatologie in vielfacher Hinsicht als Medium des Gedankenexperiments an: Sie kann als Experimentalraum dienen, um die Methoden der Primatologie und deren Ergebnisse, vor allem die Neugestaltung der Forschungsbeziehung als soziale Interaktion, zu explorieren und ihre Konsequenzen durchzuspielen, wie Goldsworthys Roman und Michael Apteds Verfilmung von Gorillas in the Mist illustrieren (Abschn. 2.5). Fiktion kann auch ein eigenes affekttheoretisches Potenzial entfalten, wenn sie sich, wie am Beispiel von Boyds Brazzaville Beach erörtert, einerseits als kathartischer Raum anbietet, in dem die Affekte der Forschenden nicht nur (wieder-)aufgeführt, sondern auch im Sinne der Destillierung eines rechten Maßes für Emotionalität abgeführt werden. Andererseits bildet Boyd in diesem Roman, ähnlich wie die frühe Primatologie mit ihrem ,Drama of Touch auf ein älteres Gefühlskonzept rekurriert, ein neues, viszerales Emotionsverständnis heraus, das die physische Symptomatik und den epistemischen Gehalt von Emotionalität aneinander koppelt und derart eine Empirie epistemischer Affektivität anbietet (Abschn. 3.5). Die literarischen Bearbeitungen primatologischer Forschung und ihrer menschlichen und tierlichen Figuren vollzieht aber auch den theoretischen und methodischen Wandel der Primatologie über die Forschendengenerationen nach. In den letzten Jahren widmet sich die Fiktion den affektiven Bedingungen der Forschung und dem Konnex dieser Affektivität mit den Gesellschaften, in denen sie stattfindet. Zudem zeigt sie sich zunehmend inspiriert von aktuellen tierethischen und -rechtlichen Debatten und elaboriert in ihrer Funktion als Verhandlungsraum die ethischen Konsequenzen von Forschungsemotionalität und einer sich wandelnden Konzeption des Tieres und seiner Rechte im Forschungskontext. Ulrike Draesner und Bettina Suleiman üben diese Auseinandersetzung der Literatur mit Ethiken der Tier-Forschung und der Emotionalität im Blick auf sich ergänzende Aspekte in Sieben Sprünge vom Rand der Welt und Auswilderung aus (Abschn. 4.5).

Es bestätigt sich bei dieser Betrachtung der primatologischen Forschungsmemoiren als Genre und der Beschäftigung fiktionaler Literatur mit der Primatologie, also an der ,Science/Fiction“ wie eingangs definiert, Sherryl Vints und Carl

\footnotetext{
${ }^{12} \mathrm{Als}$ „literature of cognitive estrangement“, so Sherryl Vint, stelle die Science Fiction einen Verfremdungseffekt dadurch her, dass ,,a novum, a new thing is introduced into the textual world that serves as the catalyst for the difference between the textual and the reader's world". Vint, Sherryl: Science Fiction. A Guide for the Perplexed. London u. a. 2015, S. 38, Hervorheb. im Original. Zur Diskussion dazu, was das derart installierte Kriterium ,alternativer Welten' für Science Fiction bedeutet, siehe Freedman: Critical Theory, S. 21.
} 
Freedmans These von der Science Fiction als kritischem Instrument, wenn nicht sogar kritischer Theorie. Kritische Theorie, so Freedman, habe als „mode of reading“ eine besondere Affinität zur Science Fiction; wichtiger aber noch: „science fiction, like critical theory, insists upon historical mutability, material reducibility, and utopian possibility. Of all genres, science fiction is thus the one most devoted to the historical concreteness and rigorous self-reflectiveness of critical theory" ${ }^{13}$ Vint elaboriert dies noch weiter hinsichtlich der Funktion von Science Fiction (und im vorliegenden Fall der ,Science/Fiction') als Medium der Sichtbarmachung:

If we think of critical theory as a set of tools and techniques that help us understand how ideology creates the world with specific values and identities, how it encourages us to understand certain things as natural and fixed and others as historical and mutable, we might think of sf as a genre that lets us see the traces of this ideological work. ${ }^{14}$

Sowohl die Forschungsmemoiren als Medium der Darstellung und Erörterung, als ,narrative Ethologie‘, als auch die fiktionale Literatur als Medium der Reflexion leisten als Konvolut der ,Science/Fiction“ eine vergleichbare Sichtbarmachung der Phänomene, Bedingungen, Theorien und Ideologien der Affekte der Forschenden, d. h. der Poetik und Politik der Emotionalität in der Primatologie.

\subsection{Affektive Teleologien}

Sei es die in den Forschungsmemoiren beschriebene, durch Anerkennungsprozesse strukturierte soziale Forschungsbeziehung zwischen Mensch(en) und Tier(en) im Feld, die durch die Literarizität der Texte gewährleistete Gestaltung der Tiere als literarische Subjekte eigener Lebensgeschichten und betrauerbare Interaktionspartner/innen oder der Wandel von Tier-Konzeptionen (wie dem animal oeconomicum) selbst: Mithilfe der kulturwissenschaftlich fundierten Lektüre der primatologischen Texte ließ sich zeigen, wie diese schon früh auf ein sich wandelndes Tier-Mensch-Verhältnis und dessen Konsequenzen verweisen, wie es etwa Donna Haraway (allerdings für ein Labor-Setting) gefordert hat: ${ }^{15}$ Wenn Forschung Arbeit ist und sie, wie in der Feldforschungssituation strukturell angelegt, nicht nur an, sondern mit Tieren unternommen wird, dann sind Tiere Arbeitssubjekte in diesen Prozessen. Wenn das Tier basierend auf der Kalkulation des Verhaltens zum eigennützigen, Investitionen in den eigenen Reproduktionserfolg tätigenden ökonomischen Akteur wird, dann unterscheidet es sich nicht mehr wesentlich von der Kategorie Mensch, deren Handeln auf Basis desselben

\footnotetext{
${ }^{13}$ Freedman: Critical Theory, S. xvi.

${ }^{14}$ Vint: Science Fiction, S. 49.

${ }^{15}$ Siehe Haraway, Donna: Becoming-with-Companions. Sharing and Response in Experimental Laboratories. In: Tom Tyler/Manuela Rossini (Hg.): Animal Encounters. Leiden 2009, S. 115136.
} 
Modells hergeleitet wird. Der Stellenwert von Mensch und Tier verändert sich vor dem Hintergrund der primatologischen Feldforschung, ihrer Methodik und der sie leitenden Denkmodelle, und vor allem durch das Medium der Forschungsmemoiren - wie die fiktionale Literatur (und der Film) schon recht schnell erkannt und exploriert hat. Dies hat Konsequenzen auch für die affektiven Teleologien der Anthropologie und Primatologie, wie sie sich am deutlichsten in der neueren Populärkultur ablagern.

Ulysse Mérou flieht am Ende des Romans La Planète des Singes vom Planeten der Affen zur Erde zurück. Ihn begleiten die sich durch Schwangerschaft und Gebären evoluierende Nova und der gemeinsame Sohn Sirius, ein ihn selbst überraschendes familiäres Glück. Doch an Bord seines Raumschiffes mischt sich in seine Erleichterung über die gelungene Flucht auch bereits ein interessanteres Sentiment: „Qui regretterai-je là-bas? Zira? Oui, Zira“(187). Zur Schimpansin Zira, seiner Be-Forscherin und später guten Freundin auf dem Planeten der Affen, hegt Ulysse eine besondere Zuneigung, und es fällt ihm schwer, diese mit den Normen seines emotionalen Regimes zu vereinbaren: „Mais le sentiment qui était né entre nous n'avait pas de nom sur la Terre ni dans aucune région du Cosmos“ (187). Die affektive Beziehung zwischen dem Menschenmann und der Schimpansenfrau darf auch auf der ,Gegenerde“ nicht sein. ${ }^{16}$ Daher ,[1]a séparation s'imposait“ (187), und um die Dinge wieder in ihre Ordnung zu setzen, wird die Wissenschaftlerin Zira sogleich noch in ein heteronormatives Gerüst eingebunden: „Elle a dû retrouver la paix en élevant des bébés chimpanzés, après avoir épousé Cornélius“ (187). Um sich selbst von der angedeuteten ,Interspecies-Romance ‘ abzulenken, versucht Ulysse Gleiches bei sich anzuwenden: „Dans notre petit univers, je ne me lasse pas d'admirer ma nouvelle famille“ (187). Aus diesem affektiven Gemisch des Abschieds zwischen emotionaler Transgression und diese einhegendem Familienglück gleitet die Erzählung Ulysses rasch zur ihn selbst in ihrer Heftigkeit überraschenden Affektivität beim Eintritt in unser Sonnensystem. „Quelle émotion“: „Des larmes me montent aux yeux. Il faut avoir vécu plus d'un an sur la planète des singes pour comprendre mon émotion..." (188).

\footnotetext{
${ }^{16}$ Die dieser Passage vorangegangene Abschiedsszene zwischen Ulysse und Zira ist dementsprechend und paradoxerweise sowohl mit Nahaffekten als auch mit dem großen Distanzierungsaffekt Ekel belegt: „,Zira!' Je me suis arrêté et l'ai prise dans mes bras. Elle est aussi bouleversée que moi. Je vois une larme couler sur son mufle, tandis que nous sommes étroitement enlacés. Ah! qu'importe cette horrible enveloppe matérielle! C'est son âme qui communie avec la mienne. Je ferme les yeux pour ne pas voir ce faciès grotesque que l'émotion enlaidit encore. Je sens son corps difforme trembler contre le mien. Je me force à appuyer ma joue contre sa joue. Nous allons nous embrasser comme deux amants, quand elle a un sursaut instinctif et me repousse avec violence.

Alors que je reste interdit, ne sachant quelle contenance prendre, elle enfouit son museau dans ses longues pattes velues, et cette hideuse guenon me déclare avec désespoir, en éclatant en sanglots. ,Mon chéri, c'est impossible. C'est dommage, mais je ne peux pas, je ne peux pas. Tu es vraiment trop affreux !'“( $P S$, S. 185, Hervorheb. MS).
} 
Doch was erwartet ihn dort, 700 Jahre nach seinem Aufbruch zur Reise ins Unbekannte, an seinem Landeplatz in Orly? Der Flugverkehr scheint ausgesetzt, der Flugplatz wirkt vernachlässigt. Ein altmodisches Fahrzeug kommt dem Raumschiff auf dem Platz entgegen. Boulle arbeitet hier mit etlichen Verzögerungen und Umschreibungen, um die Szene zu strecken, die Spannung zu steigern. Schließlich kommt der Wagen zum Halt, mehrere Personen steigen aus:

Le chauffeur est descendu. Il me tourne le dos. Il m'est à moitié caché par des hautes herbes qui me séparent de la voiture. Il tire la portière pour faire descendre le passager. Je ne m'étais pas trompé, c'est un officier; au moins un commandant; je vois briller de nombreux galons. Il a sauté à terre. Il fait quelques pas vers nous, sort des herbes et m'apparaît enfin en pleine lumière. Nova pousse un hurlement, m'arrache son fils et court se réfugier avec lui dans la chaloupe, tandis que je reste cloué sur place, incapable de faire un geste ni de proférer une parole.

C'est un gorille. (190)

Die Teleologie der Anthropologie, in der der Mensch das Endziel der zu erforschenden Entwicklung ist, wird in Planète des singes durch ein neues Evolutionsgeschehen erweitert: ${ }^{17}$ Wie auf dem Planeten Soror so auch auf der Erde wird der evoluierte Affe Mensch vom evoluierten Affen Affe als Herrschaftstier abgesetzt. Das ,Schicksal' (oder Ziel) der Menschheit wird im Roman somit durch Replikation des Experiments auch auf der Erde bestätigt und erscheint zumindest im Rahmen dieser Narration unausweichlich. Ulysse ist die Figur der Einsicht in diese Zwangsläufigkeit des menschlichen Niedergangs, die Affen sind - ihrer traditionellen epistemischen Nutzung treu - Instrumente dieser Einsicht, und die ,neue Teleologie selbst ist, wie in diesem Roman-Ende noch einmal pointiert wird, affektiv besetzt. Sie verweist gerade als solcherart Grauen erregende neue Teleologie in den 1960er Jahren auf die Umwälzungen voraus, die die Primatologie in dem hier untersuchten Jahrhundert bewirkt hat.

Die Primatologie folgte zunächst, wie einleitend erläutert, einer alten anthropologischen Teleologie. Fundamental ist ein wissenschaftliches Narrativ, in dem sich aufgrund einer mehr oder minder stringenten Evolution von Uraffe zu Gegenwartsmensch anhand der Gegenwartsaffen etwas über die biologische Herkunft und die evolutionär bedingte Sozialstruktur des Menschen erfahren lässt. Robert und Ada Yerkes beschreiben diese Voraussetzung für die Hoffnung auf Erkenntnis am Affen in ihrer psychobiologischen Überblicksstudie The Great Apes 1929 ganz affirmativ als eine zeitliche Spurensuche nach ,unserer' Vergangenheit im Affen, Schicht für Schicht:

What fascination of retrospect and revery if, to thousands of generations, one could marshal his ancestors for familiar salutation and inspection. A hundred generations pass, and one views with popping eyes forbears of Aristotelian times; another hundred, and with

\footnotetext{
${ }^{17}$ Zur Evolution als ,teleologisch auf ein bestimmtes Ziel ausgerichteter, sich in der Zeit erstreckender Ablauf" siehe Borgards, Roland: Teleologie. In: Benjamin Bühler/Stefan Willer (Hg.): Futurologien. Ordnungen des Zukunftswissens. Paderborn 2016, S. 73-83.
} 
bated breath one gazes on folk who have left scant record in artifact or rock; barely another hundred, and one's historical orientation fails. A thousand pairs pass, and the sense of understanding, sympathy, and kinship has markedly diminished. Would the procession sooner or later bring each of us face to face with a direct ancestor so apelike that he might be mistaken for, or identified with, the chimpanzee, gorilla, or orang-outan? ${ }^{18}$

Entgegen Yerkes und Yerkes' raunender Beschwörung, auf der hier niedrigsten Schicht der „procession“, beim „direct ancestor so apelike“, seien „,understanding, sympathy, and kinship" merklich verringert, ist diese Spurensuche am lebenden Affen (als Äquivalent für jenen entfernten, äffischen Vorfahren), wie hier gezeigt, eine von ,understanding, sympathy, and kinship“ ermöglichte. Es ließe sich deswegen zunächst vermuten, dass auch die Affektivität der Primatologie einer Teleologie unterliege: nicht nur einem, vom Fokus auf den epistemischen Gehalt von Emotionen in Forschungsprozessen unterstützten ,positivity-bias ' der primatologischen Affekte, der auch aus negativen emotionalen Erfahrungen noch positive wissenschaftliche Affirmation ziehen kann, sondern auch einer Entwicklung hin zu wachsender Emotionalität in der Forschung mit Affen.

Die Evolution der Primatologie spricht bezüglich der Affektregime für eine andere als diese affektive Teleologie: Wie das obig vorangestellte Zitat Wolfgang Köhlers zeigt, birgt die Gewöhnung an Affen als Forschungstiere die Gefahr der Abnutzung. Obwohl das Verständnis der sowie Sympathie und Verwandtschaft mit den Affen zunehmend durch primatologische Erkenntnisse verstärkt und in die Öffentlichkeit getragen werden, geht die Entwicklung der Primatologie und ihrer Feldforschung auch unter den sich ändernden Vorzeichen ethischer Handlungsnormen nicht unbedingt in Richtung von mehr Affektivität den Forschungstieren gegenüber. Wie sich an Volker Sommer und Julia Fischer als Autor/innen von neueren Forschungsmemoiren zeigte, führt die Konsolidierung von Forschung mit Affen im Feld unter den sich wandelnden ökologischen Bedingungen unserer Gegenwart und den ebenfalls eigenständigen Tendenzen unterliegenden Veränderungen des Forschungsfeldes zu einer Um- und Neubewertung der Affekte in der Forschung und ihrer Funktion in den berichtenden Texten. Werden Affen zunehmend öffentlichkeitswirksam und vor allem in der Fiktion zu Affektträger/innen, so scheinen sie als einzelne, affizierende Individuen aus den Forschungsmemoiren in dieser Funktion eher zu verschwinden.

Interessanterweise aber schwindet andersherum bei wachsender Popularisierung eben dieser Affen in der Populärkultur zunehmend auch der/die Feldforscher/in und mit ihr oder ihm überwiegend auch der Verdacht zoophiler ,Interspecies-Romance“ zwischen Mensch und Affe, der Ulysse und Zira am Vollzug ihrer intimen Freundschaft hinderte. Zwar erfreut sich Jane Goodall nach wie vor großer öffentlicher

\footnotetext{
${ }^{18}$ Yerkes, Robert M./Yerkes, Ada W.: The Great Apes: A Study of Anthropoid Life. New Haven 1929, S. 1.
} 
Aufmerksamkeit ${ }^{19}$ und im Feld forschende Etholog/innen fügen sich auch gut in die Expertenökonomie unserer Zeit ein. ${ }^{20}$ In den jüngsten Erzeugnissen der Populärkultur ist zudem eine Neuverhandlung der anthropologischen Differenz und vor allem der affektive Interspezies-Kontakt zu einem deutlichen, vom primatologischen Wissensfeld inspirierten ,Trend' geworden: ${ }^{21}$ In der jüngsten Verfilmung des King Kong-Stoffes, Skull Island, ${ }^{22}$ wird der Riesenaffe nicht mehr gejagt und nach New York gebracht, sondern als ,Letzter seiner Art' in seinem natürlichen Habitat auf der Totenkopfinsel zumindest zeitweilig vor dem Aussterben und der Bedrohung durch subterrane Monsterechsen gerettet. Deutlicher könnte das Naturschutzanliegen der primatologischen Rhetorik kaum umgesetzt werden.

Das ,Reboot“ des Planet-der-Affen-Stoffes, das noch einmal den Gründen für den Umsturz der primatischen Herrschaftsverhältnisse nachgeht, macht seit 2011 Affen zu zentralen Identifikationsfiguren seiner Erzählung, die nicht nur bei ihrer genetisch manipulierten ,Überaffenwerdung' begleitet werden (Rise of the Planet of the Apes), sondern auch eine (nicht ganz einwandfreie) Ethnisierung erfahren (Dawn of the Planet of the Apes, War of the Planet of the Apes), die sie zwangsläufig zu nicht immer willkommenen Interaktionspartner/innen in einem viral beeinflussten Kampf um die Vorherrschaft auf dem Planeten werden lassen. Ältere populärkulturelle filmische

\footnotetext{
${ }^{19}$ Siehe jüngst etwa ein neuer Dokumentarfilm (Morgen, Brett: Jane. National Geographic 2017), die Adelung als ,Visionary Woman' der Ökologie (Barnet, Andrea: Visionary Women. How Rachel Carson, Jane Jacobs, Jane Goodall, and Alice Waters Changed our World. New York 2018, S. 227ff.) sowie diverse journalistische Beiträge: Gerhard, Saskia: Verhaltensforschung: Drei Leben für die Affen. In: Die Zeit vom 20.03.2018, Geschichte, http://www.zeit.de/zeit-geschichte/2018/02/verhaltensforschung-affen-orang-utans-schimpanse-menschenaffen-schutz (05.04.2018); Tullis, Paul: Jane Goodall Is Still Wild at Heart. In: The New York Times vom 13.03.2015, Magazine, https://www.nytimes.com/2015/03/15/magazine/jane-goodall-is-still-wildat-heart.html (05.04.2018).

${ }^{20}$ Siehe Dinardo, Kelly: An Orangutan Expert Says Now Is the Time to Visit Indonesia. In: The New York Times vom 29.12.2016, Travel, https://www.nytimes.com/2016/12/29/travel/birutemary-galdikas-orangutan-expert-visiting-indonesia.html (05.04.2018); Rothman, Lily: Giving Names to Cute Baby Animals Can Save a Species: Jane Goodall Explains. In: Time Magazine vom 16.04.2014, Entertainment/Movies, http://time.com/64042/jane-goodall-disney-bears/ (16.05.2018). Zur Expertenkultur vgl. Schumacher, Beatrice/Busset, Thomas: ,Der Experte': Aufstieg einer Figur der Wahrheit und des Wissens. In: Traverse: Zeitschrift für Geschichte 8 (2001), S. 15-26; Thurs, Daniel Patrick: Science Talk. Changing Notions of Science in American Popular Culture. New Brunswick, NJ 2007, S. 13 f.

${ }^{21}$ In nicht-primatischen Interspecies-Plots darf auf der Leinwand tatsächlich auch noch romantisch gefühlt werden: Jüngst explorierte The Shape of Water die Zuneigung einer Menschenfrau zu einem Fischmenschen, Ex Machina interpretierte den Turing-Test wesentlich als Test der Affizierungsfähigkeit einer humanoiden artifiziellen Intelligenz und Alex Garlands Nachfolge-Film Annihilation imaginierte den körperlichen Umgang mit einer nicht-humanoiden, dafür jedoch potenziell dehumanisierenden, molekular hybridisierenden und ökologisch formenden außerirdischen Entität. Siehe del Toro, Guillermo: The Shape of Water. Fox Searchlight Pictures 2017; Garland, Alex: Ex Machina. Universal Pictures 2015; Garland, Alex: Annihilation. Netflix 2018.
}

${ }^{22}$ Vogt-Roberts, Jordan: Kong: Skull Island. Warner Bros. Pictures 2017. 
Bearbeitungen des interprimatischen Verhältnisses elaborierten dieses überwiegend als affektive intersubjektive Beziehung zwischen Affe und Mensch, indem Menschen auch als emotionale Mittler zwischen Affenprotagonist/innen und Publikum dienten. Der Roman sowie seine Verfilmung von 1968 explorierten mit der innigen Umarmung „,comme deux amants“ (PS, 185) bzw. dem Kuss zwischen Taylor und Zira ${ }^{23}$ die romantische Überschreitung der Speziesgrenze. Der Original-King-Kong umspielte mit seiner unangenehm unilateralen sexuellen Faszination von Riesenaffe für Menschenfrau ebenfalls die ,Interprimate-Romance‘. ${ }^{24}$ Der Horrorfilm Monkey Shines ${ }^{25}$ von 1988 gestaltete zwei anthropozentrische Nutzungen des Affen aus - in der medizinischen Laborforschung und im Pflege-Bereich -, wählte als Moment des heraufbeschworenen Grauens aber vor allem die primär-affektive Übertragung zwischen einem männlichen Tetraplegiker und seinem weiblichen ,Helferaffen'. Zentral ist die Eifersucht des Kapuzineraffenweibchens auf Frauen im Leben des männlichen menschlichen Protagonisten und die dadurch ausgelöste mörderische Wut. Auch Michael Crichtons Roman Congo (1995) nutzt das Element einer besonderen affektiven, heteronormativ kodierten Nähe zwischen dem die Expedition begleitenden Gorillaweibchen und ihrem betreuenden Primatologen, wenn auch ohne die sexuelle Transgression, die Goldsworthys Wish exploriert.

Seit einigen Jahren jedoch scheinen sich Affen im Film (und im Gegensatz zur hier behandelten Literatur) von ihren Menschen zu emanzipieren und ihresgleichen zu suchen. Deutlich zeigt sich das wieder am dreiteiligen ,Reboot des Planet-der-Affen-Stoffes: Zwar beginnt der erste Teil auch wieder mit einer (allerdings anders gelagerten) affektiven Beziehung zwischen Mensch und Affe: ${ }^{26}$ Der äffische Protagonist Caesar wird von einem menschlichen Forscher aus Mitleid (und ethischen Gewissensbissen) vor dem sicheren Tod gerettet und an Kindes statt aufgezogen. Auch wird Forschenden-Emotionalität im Film direkt angesprochen; so wird Will Rodman von seinem Vorgesetzten verwarnt: „Keep your personal emotions out of it". ${ }^{27}$ Die adjektivische Differenzierung (,personal“") lässt hier darauf schließen, dass die Rückkehr der Emotionalität in die Forschung bereits mitgedacht wird: Anscheinend gibt es ein Anderes dieser ,personal emotions“, und das darf ,drin bleiben“. Diese menschliche Figur Will Rodman, durch die wir an die Affen herangeführt werden, ist jedoch kein Feldforschender oder Weltraumreisender mehr, sondern ein im Dienst eines Pharmakonzerns stehender, im sterilen Labor an einer Alzheimer-Therapie arbeitender Genetiker, der - halb Ersatzvater, halb Haustierhalter - mit der Maturität des Affen Caesar weitgehend aus dem Blick verschwindet. Im zweiten Teil des

\footnotetext{
${ }^{23}$ Schaffner, Franklin: Planet of the Apes. 20th Century Fox, TC 01:44:97- 01:45:25.

${ }^{24}$ Siehe auch Lubrich, Oliver/Liebal, Katja: King Kong in the Mist. Travel, Knowledge, and Emotion. In: KulturPoetik 16/1 (2016), S. 49-63.

${ }^{25}$ Romero, George A.: Monkey Shines. Orion Pictures 1988.

${ }^{26}$ Siehe Wyatt, Rupert: Rise of the Planet of the Apes. 20th Century Fox 2011.

${ }^{27}$ Wyatt: Rise, TC 01:04:35-39.
} 
,Reboots“, Dawn, ${ }^{28}$ sind die Affen soweit durch Schmuck, Körperbemalung, Behausungen, Rituale etc. als Ethnie inszeniert, dass die menschliche Figur Malcolm, ein Architekt, sich eher in diplomatischer Mission denn als Forscher auf ihr Gebiet wagt. Im dritten Teil, War $^{29}$ sind es schließlich die Affen selbst, die gezwungenermaßen auf ,Forschungsreise ' entlang der US-amerikanischen Küste gehen und zwischen seltsam unerklärt bleibenden Anspielungen auf die Heart of Darkness-Adaption Apocalypse Now ${ }^{30}$ nach dem Verbleib der Ihren in einem Arbeits- und Konzentrationslager suchen. Menschen treten als stumme, kindliche Begleiterinnen oder als wahnsinnige Kontrahenten auf. Aber es sind die Affen selbst, die in den Worten von Caesars menschlichem Gegenspieler, The Colonel, ,so emotional“31 sind, und es sind auch die Affen, die einen ethologischen Blick auf die Menschen wenden: Im Zentrum steht das Verhalten der Menschen angesichts der Evolution und Verbreitung der Affen und ihres eigenen krankheitsbedingten Niedergangs. Die Affen beobachten sie dabei: unter Zuhilfenahme eines Feldstechers, mit bloßem Auge aus der Ferne und anhand von Spuren und ,Hinterlassenschaften“, aus der Höhe, selbst aus dem Käfig heraus noch. Als solcherart Beobachtende der menschlichen Apokalypse werden die äffischen Protagonisten geradezu in Szene gesetzt; in War sehen wir bspw. mit dem Kamerablick zunächst über die Schulter des Bad Ape und zum Schluss über jene des Protagonisten Caesar mit diesen Affektträgern den Menschen bei der Selbstzerstörung zu. ${ }^{32}$

Diese Entwicklung des Stoffes kann auf zweierlei verweisen: Zum einen scheint die populäre Fiktion anzunehmen, dass, solange der rechtlich-taxonomische Status der (Menschen-)Affen ungeklärt bleibt und daher vertraute ethische Normen infrage stehen, die Forschung mit Affen zwischen Ethologie und Ethnologie und affektive Beziehungen mit ihnen zwischen Interspezies- und interkultureller Romantik gefangen und damit für das Mainstream-Kino zu heikel sind. Zum anderen liest die Populärfiktion die Konsequenz des basierend auf der Feldforschungsarbeit der Primatologie betriebenen ,Selfing the Ape' als Emanzipierung des Affen vom Bann eines menschlich vermittelten und interpretierenden Blicks. Der primatologische Beobachter/innen-Blick, wie ihn die Forschungsmemoiren vermittelt und tradiert haben und die Literatur analysiert und elaboriert hat, scheint sich in diesen Filmen in dem Maße aufzulösen, wie die Affen anthropomorphisiert werden. Damit reflektiert die Fiktion eine zwangsläufig anmutende Entwicklung des Faches: Denn die emotionale Humanisierung des Affen bei gleichzeitiger Primatisierung des Menschen lässt die Primatologie als Teil der Anthropologie hinter sich. Aus der Entwicklung hin zur

\footnotetext{
${ }^{28}$ Reeves, Matt: Dawn of the Planet of the Apes. 20th Century Fox 2014.

${ }^{29}$ Reeves, Matt: War of the Planet of the Apes. 20th Century Fox 2017.

${ }^{30}$ Coppola, Francis: Apocalypse Now. United Artists 1979.

${ }^{31}$ Reeves: War, TC 01:24:07-09.

${ }^{32} \mathrm{Vgl}$. Reeves: War, TC 00:35:09-30 und 01:14-51-15:17; 00:39:53-41:10; 00:56:15-20; 01:07:11-33 und 01:35:01-50; 01:52:33-45; 02:03:12-04:12.
} 
Anthropologie als vergleichende Primatologie muss schließlich der Versuch resultieren, den menschlichen Blick nachhaltig zu dezentrieren und vielleicht sogar zu transzendieren. Auf dem Planeten der Affen blicken die Affen nicht allein zurück; sie beobachten Menschen dabei, wie sie emphatisch emotional (wieder) Affen werden.

Open Access Dieses Kapitel wird unter der Creative Commons Namensnennung 4.0 International Lizenz (http://creativecommons.org/licenses/by/4.0/deed.de) veröffentlicht, welche die Nutzung, Vervielfältigung, Bearbeitung, Verbreitung und Wiedergabe in jeglichem Medium und Format erlaubt, sofern Sie den/die ursprünglichen Autor(en) und die Quelle ordnungsgemäß nennen, einen Link zur Creative Commons Lizenz beifügen und angeben, ob Änderungen vorgenommen wurden.

Die in diesem Kapitel enthaltenen Bilder und sonstiges Drittmaterial unterliegen ebenfalls der genannten Creative Commons Lizenz, sofern sich aus der Abbildungslegende nichts anderes ergibt. Sofern das betreffende Material nicht unter der genannten Creative Commons Lizenz steht und die betreffende Handlung nicht nach gesetzlichen Vorschriften erlaubt ist, ist für die oben aufgeführten Weiterverwendungen des Materials die Einwilligung des jeweiligen Rechteinhabers einzuholen. 